\title{
Analysis on the Relation between Software Development Pace and Network Traffic Pace as a Consequence of Rapid Business Development
}

\author{
Dr. W.U.Khan \\ Professor, Dept. of CSE \\ SGSITS, Indore, INDIA.
}

\author{
Majid Ahmad \\ Asst.Proffesor, Dept. of MCA \\ MITM Indore, INDIA.
}

\begin{abstract}
This paper acknowledges the concern over mild network bandwidth advancements as compared to the alarming traffic induced due to latest $4 \mathrm{th}$ generation (Rapid development) tools for software development. The growing web based commerce is one of the main reasons that induced the pressure on the software industries to come up with the magical speedy development tool kits. The web now very much overloaded with this all, has a direct impact on the traffic over wired/wireless lanes. The system (communication lines) for the running traffic has been same but with of course overhauled for some gain in speed and efficiency (bandwidth). The proportion with which the traffic is increasing on the web is on the contrast to the breakthrough the researcher's are getting in bandwidth enhancements. Although we are providing the answer by adding more bandwidth, but that isn't a long lasting solution. The ratio is of quite concern if not now but in near future. In this paper we register our first impression towards this concern, as we are presenting a comparison between the increased traffic and the scarce network resource (Bandwidth).
\end{abstract}

\section{General Terms}

Web Analysis, Software development Analysis, Communication Bandwidth.

\section{Keywords}

Bandwidth, Web-Traffic, Website-classification.

\section{INTRODUCTION}

Software has extended many areas of modern life. The usages of the software in past were generally confined to areas and applications like simple word processors, play games, online military control equipments and general data transfer across computers. In recent years, the applications of software have dominated many fields of our life. One of the most important area being the webbased commerce, that has given the software industry a promising yet elevated career ahead at least for few decades or so[4].

Almost every other field of science and development does have an impact on the software development in large [5].The development of these especially web based software products are being built to provide the customers with every functionality they intend / desire to have for the operational functioning of there concerned business. The advent of ecommerce (m-banking, online shopping, shipment tracking, online-invoice etc) has provided challenges to the software industry to deal with. The desires of human (customers) don't have a limit as such, and the developer's are equally tossed up to craft there desires to pragmatic products. Although it was being felt back in late 1960's, but software started to play a major role in market trends only after 1990's [2]. The introduction of these software products (web based) is providing the sky-scraping heights to the day to day commerce at least in two aspects. Firstly, as a latest market trend for the current competitive trade. Secondly, providing an ease to bussiness customers for marketing and customer support. The above all reasons provided enough spur for the software developing industry to come up with some highly sophisticated development environments, that will result in rapid software production that too with highend services[1]. RFID technology and the other Network Solutions have attracted growing interest from a wide range of industries and research academics [9]. The fourth generation techniques for software development especially web-based stared a new era of software development. The use of these tools made the software industry to take the business challenges with more composure and buoyancy [3]. The shortcomings with most of these visual development tools is that they have extended the human comprehension far too much that leads to increased size of the final deliverable products.

\section{BACKGROUND SURVEY}

As Netscape cofounder Marc Andreessen famously termed the operating system as a," poorly debugged set of device drivers". Because of increasingly interactive online code, the near future will replace the desktop with the WebTop. The WebTop is almost on cards as of now. The websites development rate is far from the assumed as not only urban but also rural areas have the online services. The backbone to them being the rapid application development tools. The concept of $4 \mathrm{gl}$ technique came from various sources and reasons. The important cause if being the rapid development, but still to the popularity of these visual rapid tools came because of some other reasons too. Ease of use( The approach of $4 \mathrm{GLs}$ is strictly application oriented. The concept of dealing with implementation related difficulties was removed from the 
developer's mind. Thus resulting in fast and impressive development). Advanced embedded functionalities (4GLs are typically accompanied by a set of functions or specific applications. This is because the product then becomes easier to use than a full programming language). Testing tools (Higher level languages often restrict the options available to developers of lower level languages, such as the capability to modify code structure at execution time. To compensate for this, 4GLs permit automatic verification before testing. With 4GLs, a dialogue occurs between the developer and the computer. So fast errors tracking, even at the time they are being made. Almost the developer no longer needs to be syntax conscious). Miscellaneous (A user of a 4GL is not required to specify everything, Instead a compiler or interpreter is capable of making intelligent assumptions about the user needs. Therefore, while 4GLs often require that many parameters be specified, they also provide a default option if the user does not make a selection. This saves time and debugging efforts). As the above all elements were taken as a well beginning to the speedy development, but the more advancement of each of them started to build a sense of load to development environment. Thus the load can be felt both ways: Developer load and product load while the developer load can be defined as the amount of anxiety towards the software development. The factors accounting to the anxiety can be (Lines of code, time of development, syntax sensitivity, approaching deadline, Bug tracking etc). The introduction of the 4GL techniques led to lessen the above anxiety affecting effects. The product load is size of the product (the amount of disk space it takes). The development in a highly sophisticated environment adds up so much of additional size that it ends up in large overhead. According to previous and current trend, we can depict the load factor related to time in conjunction with product and developer. Figure 1 shows the scenario which is more of a heuristic or metaphor than an iron-clad empirical derivation. There is a knee curve for the developer load as it is keeping a constant trend after a sharp decrease. On the contrary the product load has got a climbing curve. The above graph can be stated as valid, if taking into consideration the trend from assembly to high-level languages. On the other hand, the bandwidth advancement is having a trend of some burst success at times, but the some issues always limit the maximum achievable bandwidth(achievable throughput). Here we enlist a few of the issues: Hannon-Hartley theorem, skin effect, parasitic capacitance, Free-space loss and many more. Electronic industry has provided us the traffic measurement tools for internet. Most recently the Comcast, Inc. has launched a usage meter for internet subscribers. The meter has been tested thoroughly by NetForecast, Inc. The Comcast usage meter is believed to be robust and is expected to provide the usage information to the subscribers up to some standard accuracy. Thus the industry is taking steps to regulate the traffic if not at provider's level, but at least at user level. These regulatory measures in future will be more helpful to monitor the traffic.

\section{ANALYSIS}

To help a better tradeoff for the development pace and network traffic a more practical approach was taken to do the analysis. The Survey was done on different available websites, mostly in concern with the business/commerce. The Business websites mostly were chosen from a varied group of business areas. The effectiveness of the website is a major factor to be taken into consideration [8]. The environments chosen were HTML, PHP, ASP(.Net).

Few assumptions being made.

1. "An equal, finite number of businesses sites were selected from each business area".

2. "Any business site can be classified into one of the three groups. The groups being made on the basis of availed functionality namely (Basic, medium, Extended)".

3. "After having three final groups. Each of the sites in every group was developed in three scripting environments".

The web based surveys in past have been done for social and regional information study [5]. The present survey started with intent to verify our belief that, the rapid business developments adds much to the traffic enhancement on the communication networks, as a consequence of usage of the $4 \mathrm{gl}$-languages (rapid developmental tools). As our initial survey, we found the results up to our expectations. The results as evident from the figure 2.a are showing an upward trend as the development tools get more automated and sophisticated, that results in sharp increase in size. As we go on adding the functionalities the size of the site increases exponentially. Thus the high-end functionalities demand much space and in turn increase the network traffic drastically. Figure2.b shows us the effect of developmental environments. The development in ASP (.Net) is always consuming more size irrespective of site-category. While as the development in HTML is nominal as compared to the others (ASP,PHP).

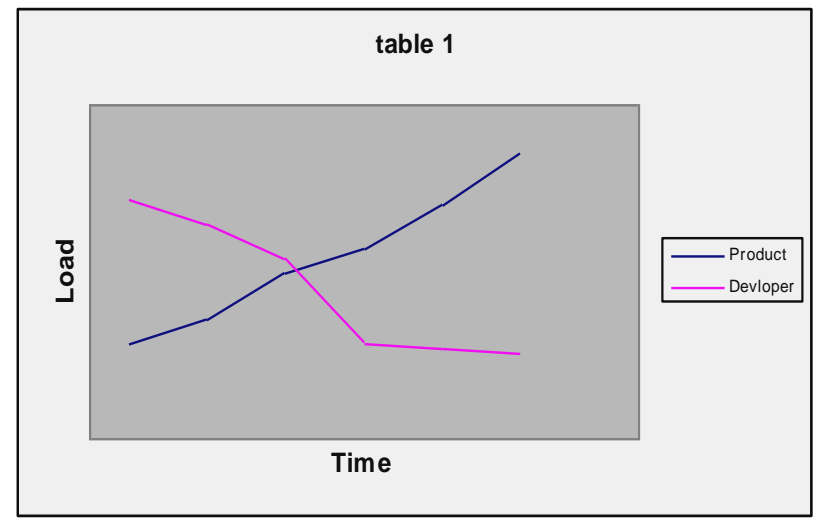

Figure 1: Time Vs Load

\section{CONCLUSION}

We have presented a Survey that provides us an insight about the role of rapid visual developmental tools on increasing the traffic on the available bandwidths. Thus more speedy software development if on one hand helps to meet both the short deadlines and the production load of software industry, but to the concern of communication industry it does provide 
some tough challenges ahead

\section{FUTURE WORK}

Our study on the rapid development environments is continuing. We suggest that other parameters other than bandwidth can also be analyzed to asses the trend due to rapid developmental tool usage.

\section{RESULT SET DISCUSSION}

The analysis can be summed up and presented in the following figures. The various parameters present more lucid sight about the findings of our work. Figure 2.a shows us that the websites belonging to basic category don't have any severe effect on the final product size, while we move from basic to extended (functionalities availed) the size increases exponentially. As the more advancements and introduction of high end services are proving as market driver (agent), so business these days are bound to have extended class of websites. Figure 2.b on the other hand is another way to deal with our findings to keep a check on website development by fourth-generation language tools. The more introduction of these tools are making servers overloaded consequently making the web traffic towering.

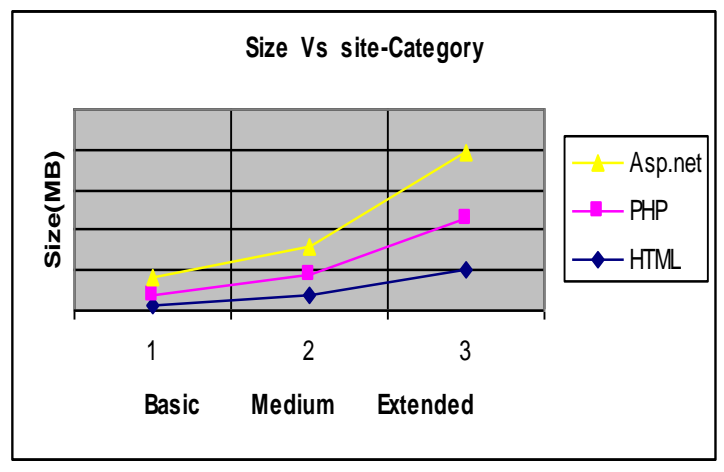

Figure 2.a

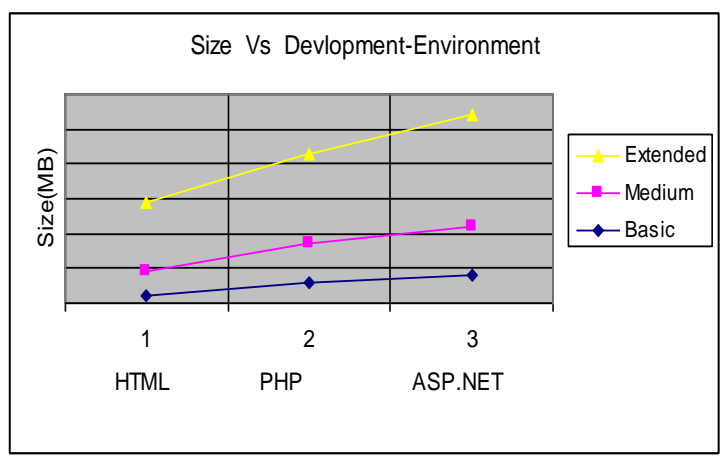

Figure 2.b

\section{REFRENCES}

[1] "What is Rapid Application Development?" @Copyright 1997-2000CASEMakerInc.

[2] "Process-Centered Software Engineering Environment" Garg, P. K. and M. Jazayeri (eds.), IEEE Computer Society, pp. 131-140,1998.

[3] "Application Development Without Programmers." Martin Prentice-Hall, 1981. ISBN 0-13-038943-.

[4] “ A survey of web engineering practice in small Jordanian web development firms", Proceedings of the 6th joint meeting of the European software engineering conference and the ACM SIGSOFT symposium on The foundations of software engineering.

[5] "Conducting web-based surveys. Practical Assessment, Research \& Evaluation”, Solomon, David J. (2001).

[6] "A Comparative Analysis of Software Engineering with Knowledge Engineering", J. Frank Vijay and C. Manoharan, India, Journal of Computer Science 6 (10): 1208-1211, 2010 ISSN 1549-3636 (C) 2010 Science Publications.

[7] "Geospatial Cyber infrastructure: Past, Present and future "

Computers, Environment and Urban Systems, Volume 34, Issue 4, July2010, Yang, C.; Raskin, R.; Goodchild, M.; Gahegan, $M$.

[8] "Explaining website effectiveness: The hedonicutilitarian dual mediation hypothesis "Electronic commerce research and applications, Volume 10, Issue1, January-February 2011, Pages 49-58 Special Section: Service Innovation in E-Commerce, Inés Lópezaand.Salvador.Ruiz.

[9] "Business process analysis and simulation for the RFID and EPCglobal Network enabled supply chain: A proofof-concept approach", Network and Computer Applications, Volume 34, Issue 3, May 2011, Worapot Jakkhupan, Somjit Arch-int and Yuefeng Li 